Friedrich F. Hennig

Unfallchirurgische Abteilung, Universitätsklinikum Erlangen

\title{
Posttraumatische Gonarthrose, Teil 1
}

\section{Zusammenfassungen der Vorträge}

\section{Richtungsweisende Diagnostik bei posttraumatischen Zustän- den (W. Mittelmeier, München)}

Bei der Diagnostik posttraumatischer Zustände am Kniegelenk kommen nahezu alle Bild gebenden Verfahren zum Einsatz. Der diagnostische Einstieg sind die konventionelle Röntgendarstellung des Kniegelenks und eine Ganzbeinaufnahme als so genannter Bein-BeckenStatus. Diese Aufnahme sollte unter Körperbelastung im aufrechten Stand angefertigt werden, da nur so die indirekte Beurteilung der Knorpelqualität der Gleitflächen möglich ist.

Die kernspintomographische Aufnahme schließt sich als nächster differenzierender diagnostischer Schritt an. Durch die verschiedenen Frequenzeinstellungen kann eine sehr aussagekräftige Diagnostik betrieben werden, die auf dem Gebiet der Kapsel-Band-Schädigung sowie der Knorpelbeurteilung Aussagen mit hoher Sicherheit erlaubt.

Die moderne Technik der Kernspintomographie birgt jedoch auch eine Gefahr. Die große Frequenzbreite, die zum Einsatz kommen kann, erfordert eine differenzierte Beurteilung, die ohne eine differenzierte Erfahrung nicht möglich ist. Hier müssen Standardfrequenzen bezüglich ihrer Aussagekraft erarbeitet und eingehalten werden, um nicht nur dem ausschließlich in der Diagnostik tätigen Arzt, sondern auch dem therapeutischen Arzt ein sicheres und reproduzierbares Beurteilungsinstrument an die Hand zu geben. Nur so ist es möglich, früh- und spätposttraumati- sche Zustände bezüglich Therapie und Prognose richtig zu beurteilen.

Zukünftige diagnostische Techniken, wie die Fluoreszenzradiographie, müssen sich noch im klinischen Alltag bewähren. Die Aussagekraft der Szintigraphie ist für die Beurteilung posttraumatischer Zustände zu vage.

\section{Knorpel-Knochen-Transplan- tation - Was ist gesichert?$$
\text { (J.H. Lill, Leipzig) }
$$

Die Knochen-Knorpel-Transplantation ist ein klinisch validiertes Therapieverfahren mit guter Erfolgschance bei richtiger Indikation. Sind posttraumatische Achsfehlstellungen mit Überbelastung des geschädigten Knochen-KnorpelAreals ausgeschlossen, kann die Transplantation eines mit einem Spezialinstrumentarium passgenau ausgeschnitten Knorpel-Knochen-Zylinders aus unbelasteten Zonen in die geschädigte Kniegelenkzone mit guten bis sehr guten Ergebnissen aufwarten.

Die Methode wurde bisher überwiegend in offener Technik durchgeführt. Diese Technik ist bei großem Defekt auch weiterhin erforderlich. Die Transplantation von mehreren Zylindern groBen Durchmessers gelingt nur durch dieses Operationsverfahren. Bei umschriebenem kleinerem Defekt bewährt sich zunehmend die arthroskopische Knochen-Knorpel-Transplantation.

Trotz gewisser Umstrukturierung des transplantierten Knorpels ist die Knorpelqualität hochwertig. Röntgenologische, arthroskopische und biopti- sche Untersuchungen sowie die klinische Erfahrung haben dies bestätigt. Bei großen Defekten wird die Transplantation als so genannte Mosaikplastik durchgeführt. Als Empfängerareale kommen der Femurkondylus, die patellare Gleitfläche und die tibiale Gleitfläche in Frage. Neben der oben bereits erwähnten korrekten Achsverhältnisse im Kniegelenk ist besonders auch im Bereich der Kniescheibe auf eine ligamentäre Balance zu achten.

Bei richtiger Indikation und korrekter Operationstechnik ist eine frühfunktionelle Nachbehandlung mit zügigem Übergang zur Belastung empfehlenswert.

\section{Therapiemöglichkeiten der retropatellaren Arthrose (R. Ascherl, Leipzig)}

Die Funktion der Kniescheibe muss vor dem Hintergrund der Beinachse, der ligamentären Balance und der muskulären Balance gesehen werden. Anlagebedingte Form und Formvarianten der Kniescheibe spielen eine deutlich nachrangige Rolle. Korrektureingriffe an der Kniescheibe zur Veränderung ihrer vor-

\footnotetext{
C) Springer-Verlag 2003

Prof. Dr. Friedrich F. Hennig Unfallchirurgische Abteilung, Universitätsklinikum Erlangen, Krankenhausstraße 12,91054 Erlangen, E-Mail:unfall@chir.med.uni-erlangen.de, Tel.:09131-8533272, Fax:09131-8533300
} 
gegebenen Form sind überwiegend verlassen.

Vor dem Hintergrund dieser Aussage ergeben sich die Therapieansätze. Achsfehlstellungen im Kniegelenk sind unter Berücksichtigung der Beinachse und der Kniebasislinie zu korrigieren.

Ein laterales Hyperpressionssyndrom ist in erster Linie durch intensive medikophysikalische Maßnahmen im Sinn des gezielten Muskeltrainings des Vastus medialis zu therapieren. Ergänzend kann eine Retinaculumplastik erforderlich sein. Diese besteht jedoch nicht im alleinigen Schlitzen des Retinaculums, sondern in einem chirurgisch korrekten Release und einer straffenden Plastik im Bereich des medialen Retinaculums.

Nur vereinzelt, bei therapieresistenten Fällen und anlagebedingtem, exzentrisch lateralem Ansatz des Lig. patellae an der Tuberositas tibiae, sind das Ablösen von bis zur Hälfte des lateralen Lig. patellae von der Tuberositas tibiae und Medialisieren des Bands durch Unterschlagen des Restbands und Refixation im Periost erforderlich. In ausgeprägten Fällen des Fehlansatzes können die Medialisierung, ggf. auch die diskrete Ventralisierung des knöchernen Lig.-patellae-Ansatzes durch Knochenblocktransposition erforderlich sein. Dies bedarf jedoch des Hinweises an den Patienten, dass hierdurch das Knien auf dem Kniegelenk nach stattgehabtem Eingriff be- schwerdefrei, zumindest für eine sehr lange Zeit, nicht mehr möglich ist.

In der Zusammenfassung wies der Referent nachdrücklich darauf hin, dass die retropatellaren Beschwerden in Folge einer retropatellaren Arthrose primär ein Gebiet der konservativen medikophysikalischen Therapie sind und nur in wenigen berechtigten Fällen einer chirurgischen Therapie zugeführt werden müssen. Ein alleiniger retropatellarer alloarthroplastischer Gleitflächenersatz muss völlig abgelehnt werden, da dieses Vorgehen nicht zum Erfolg führt.

\section{Umstellungsosteotomie bei Gonarthrose (C. Josten, Leipzig)}

Die Umstellungsosteotomie zur Behandlung der Gonarthrose kommt zum Einsatz, wenn angeborene oder erworbene Achsfehlstellung das Kniegelenk einseitig belastet haben. Das therapeutische Ziel ist die Entlastung des geschädigten Kompartments durch Wiederherstellung der achsgerechten physiologischen Belastung.

Fällt die Entscheidung zu dieser Maßnahme rechtzeitig und ist die Schädigung im überbelasteten Kompartment noch nicht zu weit fortgeschritten, kann v. a. bei jüngeren Patienten eine deutliche Erholung des geschädigten Knorpels im postoperativ entlasteten Kompartment beobachtet werden.

Auch wenn dieser Prozess im höheren Alter weniger wahrscheinlich ist, stellt allein die mechanische Entlastung eine erhebliche Beschwerdelinderung für den Patienten dar. Das Fortschreiten der Gonarthrose kann zumindest deutlich verzögert werden.

Die Umstellungsosteotomie erfordert stets eine komplexe präoperative Bild gebende Diagnostik. Die Entscheidung für eine arthroskopische Beurteilung des Kniebinnenraums sollte großzügig gestellt werden. Die Auswahl der verschiedenen Osteotomieverfahren zur Achskorrektur wird durch die Fehlstellung und den Ort der Fehlstellung bestimmt. Nur ein Operateur, der die gesamte Palette der subtraktiven und additiven Osteotomieverfahren sowie die gesamte Palette der stabilisierenden osteosynthetischen Verfahren beherrscht, kann hier erfolgreich tätig sein. Die Achse muss im erforderlichen Umfang und am richtigen Ort sowie in der richtigen Höhe korrigiert werden. Eine lastlinienkorrekte Position des Kniegelenks und eine achsgerechte Stellung der Kniebasislinie müssen angestrebt werden. Durch interligamentäre Umstellungen kniegelenknah können Bandlaxizitäten erforderlichenfalls mit korrigiert werden.

Trotz der Komplexizität des erforderlichen Eingriffs sind, soweit möglich, Kriterien der minimalen Invasivität $\mathrm{zu}$ berücksichtigen. Übermäßig große operative Zugänge stören die Funktionalität der Gleitschichten, vor allen Dingen kniegelenknah, und erhöhen das Risiko bei nicht selten später erforderlichen endoprothetischen Eingriffen. 